Itinéraires Itinéraires

Littérature, textes, cultures

\title{
Michael Levenson (dir.), The Cambridge Companion to Modernism
}

\section{Aurore Touya}

\section{OpenEdition}

\section{Journals}

Édition électronique

URL : http://journals.openedition.org/itineraires/550

DOI : 10.4000/itineraires.550

ISSN : 2427-920X

Éditeur

Pléiade

\section{Édition imprimée}

Date de publication : 1 novembre 2009

Pagination : 174-176

ISBN : 978-2-296-10115-9

ISSN : 2100-1340

\section{Référence électronique}

Aurore Touya, « Michael Levenson (dir.), The Cambridge Companion to Modernism », Itinéraires [En ligne], 2009-3 | 2009, mis en ligne le 26 juin 2014, consulté le 21 décembre 2020. URL : http://

journals.openedition.org/itineraires/550 ; DOI : https://doi.org/10.4000/itineraires.550

\section{(c) (1) $\odot$}

Itinéraires est mis à disposition selon les termes de la licence Creative Commons Attribution - Pas d'Utilisation Commerciale - Pas de Modification 4.0 International. 


\section{Michael Levenson (dir.), The Cambridge Companion to Modernism, Cambridge University Press, 1999.}

Tous les auteurs ayant collaboré à l'ouvrage dirigé par Michael Levenson soulignent d'emblée le caractère mouvant, approximatif de la notion de modernism: l'introduction, qui fait coïncider le modernism avec les trente premières années du $\mathrm{XX}^{\mathrm{e}}$ siècle, semble démentir la chronologie qui l'a précédée, qui rappelle les dates de création des grandes œuvres modernistes sur une période allant de 1890 à 1939.

Cette première contradiction apparente est bien à l'image des difficultés inhérentes à cette notion : le modernism, du fait de ses liens étroits avec ce qui l'a précédé - événements historiques, soubassements métaphysiques et créations artistiques de la fin du XIX ${ }^{\mathrm{e}}$ siècle - et ses héritages - notamment en matière de création littéraire, dramatique et picturale - est difficile à isoler.

Mais cette difficulté ne doit pas minimiser pour autant l'importance de ce mouvement culturel, bien au contraire. Situé au centre de questionnements philosophiques et artistiques, et à une charnière historique, le modernism est une notion clé de l'histoire artistique et littéraire du $\mathrm{XX}^{\mathrm{e}}$ siècle : c'est bien ce que s'attachent à montrer les différents collaborateurs de cet ouvrage.

Si l'on croise les articles qui constituent cette étude, il apparaît que le modernism est un courant de pensée qui traverse les différents champs de la création artistique du début $\mathrm{du} \mathrm{xx}^{\mathrm{e}}$ siècle. Certes, ce courant semble à certains égards dépasser ses propres bornes chronologiques, et quelques détours sont nécessaires aux auteurs afin d'éclairer les soubassements et les conséquences du modernism; néanmoins, le début de la Seconde Guerre mondiale marque un repère final clair.

Que se passe-t-il donc durant cette période? Qui sont ces « modernistes », et où exercent-ils leur mode de pensée?

Au début $\mathrm{du} \mathrm{xx}^{\mathrm{e}}$ siècle, la pensée et la création modernistes se développent dans différents champs de la société anglo-saxonne, puis occidentale dans son ensemble: c'est bien ce qu'illustre le mode de construction de cet ouvrage, qui propose d'étudier dans chaque article le modernism sous un de ses aspects particuliers, dans un domaine artistique et/ou générique distinct.

Ce qui rapproche les artistes et les penseurs modernistes, ainsi que le souligne Michael Bell dès le premier article de l'ouvrage, qui porte sur la métaphysique du modernism, c'est un même intérêt pour la question du lien entre la forme que prend l'œuvre d'art et les modes de connaissance ou de compréhension du monde et de la société. Pour les modernistes, différentes conceptions du monde peuvent cohabiter, sans que l'une d'elles ne prime absolument sur les autres : la création artistique, en fonction de la forme qu'elle prend, reflète une vision du monde singulière.

Cet intérêt commun permet donc de rapprocher des domaines et des pratiques aussi distincts que ceux qui composent l'ensemble du champ de la 
création artistique, qu'elle soit littéraire (elle-même constituée des genres de la fiction romanesque, de la poésie et du théâtre) ou visuelle (regroupant la peinture, la sculpture, l'architecture et le cinéma). À la création artistique s'ajoutent les professions intellectuelles, elles aussi traversées en ce début de siècle par la pensée moderniste, et que l'on peut diviser en deux parties : d'une part les milieux de l'édition, le marché de l'art et le mécénat, et de l'autre le monde académique, puisque la pensée moderniste trouve parfois dans l'université une caisse de résonance. Enfin, la pensée moderniste essaime jusque dans le domaine politique, où elle exerce une influence sur des individus appartenant à plusieurs types de partis (de la gauche à l'extrême droite), ainsi que sur des mouvements sociaux récemment constitués et qui veillent à promouvoir certaines minorités jusque-là négligées (les femmes, les Noirs).

Comment expliquer alors que la pensée moderniste trouve un tel écho dans des champs si différents? Il faut rappeler que de nouvelles questions sociales et politiques agitent la fin du XIX ${ }^{\mathrm{e}}$ et le début du $\mathrm{XX}^{\mathrm{e}}$ siècle, et font de cette période un temps de crise et de doute : les artistes sont certainement enclins à accueillir de nouveaux modes de pensée et de représentation, qui leur semblent davantage en accord avec la réalité de leur temps.

La période moderniste est évidemment marquée par le premier conflit mondial, qui marque en profondeur les mentalités, et par l'ouverture d'un second conflit à la fin des années trente, encore plus mondialisé. La violence de la guerre se retrouve sans nul doute dans certaines œuvres modernistes, qui, par un phénomène mimétique, reflètent la souffrance occasionnée, comme le souligne James Longenbach dans son étude sur la poésie moderne. À l'horreur de la guerre s'ajoutent les combats sociaux pour l'égalité des membres de la société quel que soit leur sexe - c'est l'objet de l'étude de Marianne Dekoven, intitulée «Modernism et genre »- ou leur couleur de peau - comme le montre l'article de Sara Blair sur le lien entre le modernism et les politiques culturelles.

Ce contexte historique particulier permet donc d'établir dans les mentalités un lien entre ce qu'est devenue la société moderne et le champ d'action de l'artiste : les créations modernistes reflètent les modifications de la société, en même temps qu'elles sont pour les artistes un moyen d'agir, de s'engager ou simplement de se prononcer sur le monde qui les entoure.

À cela s'ajoutent les profondes modifications métaphysiques liées au modernism, dans ces années que l'on a parfois regroupées sous le terme d'ère du soupçon, marquées par un scepticisme herméneutique, héritage de la pensée de Nietzsche et de Marx : depuis le travail de Freud entamé dès la fin du XIX siècle, l'importance de la subjectivité de chacun est reconnue, et la notion d'inconscient prend une place grandissante, en particulier dans le domaine des arts visuels modernes, qui donnent naissance à de nouveaux mouvements tels que le cubisme, le dadaïsme et surtout le surréalisme, ainsi que le montre l'article de Glen Macleod. Cette étude illustrée de reproductions souligne les liens qui unissent étroitement l'art pictural et la littérature moderniste : la peinture est en effet, dès le XIX ${ }^{\mathrm{e}}$ siècle, la forme d'art la plus novatrice, qui travaille à explorer les possibilités offertes par 
les supports et s'oppose à l'académisme et à la normativité des salons. Cet intérêt des peintres pour les nouveaux types de représentation, qui passe entre autres par l'impressionnisme et l'abstraction, est très proche de celui des romanciers modernistes, dont Woolf, Joyce et Faulkner sont les figures de proue : les auteurs de fiction du début $\mathrm{du} \mathrm{xx}^{\mathrm{e}}$ siècle ressentent eux aussi la nécessité de recourir à de nouvelles formes de récit. Le roman, qui a été durant les périodes victorienne et edwardienne un genre très prisé, doit se renouveler s'il veut parvenir à dire quelque chose de la réalité du siècle naissant. Les romanciers modernistes sentent que le monde imaginaire du roman edwardien ne correspond plus au monde qui les entoure : ils travaillent dès lors à trouver une nouvelle fiction adéquate, dont la forme aussi puisse être l'image de la complexité de la vie. À ce titre, il faut rapprocher l'article de David Trotter consacré au « roman moderniste » de celui de Lawrence Rainey qui étudie l'économie culturelle du modernism : l'exemple de la publication d'Ulysses, de l'Irlandais James Joyce, par Silvia Beach en 1922 illustre bien l'affranchissement de la littérature moderniste, qui fait fi des normes génériques et n'hésite pas à s'opposer aux censeurs.

Enfin, la pensée moderniste trouve un écho tout particulier dans les domaines de la création théâtrale et cinématographique, champs qui sont traités séparément dans l'ouvrage, mais qui présentent un certain nombre de points communs. Ces deux formes d'art visuel sont étudiées sur des périodes excédant le cadre historique préalablement déterminé : si le cinéma naît à la fin du XIX ${ }^{\mathrm{e}}$ siècle, il faut, nous dit Michael Wood dans son article, attendre les années 1930 pour que la sonorisation des films soit généralisée, et que la pratique du montage soit analysée et assimilée à une syntaxe narrative qui souligne à son tour l'importance de la forme dans la création. Le modernism dramatique quant à lui, est toujours à l'œuvre dans le théâtre d'aujourd'hui : Christopher Innes établit une filiation entre les pièces de Strindberg, de Brecht et de Pinter, et va jusqu'à voir dans le modernism une des normes de la dramaturgie contemporaine.

Ainsi, l'ouvrage dirigé par Michal Levenson est une vaste investigation, menée dans tous les champs concernés, sur la place de la pensée moderniste chez les artistes et les penseurs du début du $\mathrm{xx}^{\mathrm{e}}$ siècle. Chaque article propose une étude détaillée et spécifique, s'appuyant sur des exemples précis, qui permettent au lecteur d'affiner sa connaissance de l'œuvre des grands artistes modernistes, tout en comprenant le lien de chacun avec un courant de pensée général. Certes, tout ne peut être dit dans un article, et l'on regrette parfois que la notion centrale de modernism semble encore se refuser à certaines définitions qui permettraient de classer définitivement un artiste ou son œuvre. Mais sans doute est-ce là aussi ce qui fait la force du modernism, que d'être un courant de pensée fortement lié à ce qui l'a précédé, et qui irrigue toujours, jusqu'à aujourd'hui, certains questionnements esthétiques partagés par les différentes catégories de l'art moderne. 